\title{
Clima de segurança em centro cirúrgico: validação de um questionário para o cenário brasileiro
}

Daniela Campos de Andrade Lourenção ${ }^{1}$,

Daisy Maria Rizatto Tronchin²

\section{RESUMO}

Validar a versão traduzida e adaptada culturalmente do Safety Attitudes Questionnaire/Operating Room Version para o contexto brasileiro. Estudo metodológico de validação das propriedades psicométricas do questionário adaptado para o contexto brasileiro. A amostra correspondeu a 590 profissionais de saúde atuantes em centro cirúrgico, de quatro hospitais brasileiros, coletando-se os dados de maio a agosto de 2014. A validação de constructo ocorreu pela análise fatorial exploratória e a confiabilidade pelo alpha de Cronbach. A análise originou um questionário com 40 assertivas alocadas em seis domínios: clima de segurança, percepção da gerência, percepção do estresse, condição do trabalho, comunicação no ambiente cirúrgico e percepção do desempenho profissional. A versão adaptada mostrou-se confiável, obtendo o valor geral do alpha de Cronbach de 0,87 e entre os domínios, o alpha variou de 0,59 a 0,87. Esta pesquisa permitiu validar um instrumento passível de avaliar o clima de segurança em centro cirúrgico.

Descritores: Segurança do Paciente; Centros Cirúrgicos; Enfermagem Perioperatória; Cultura Organizacional; Estudos de Validação.

\footnotetext{
${ }^{1}$ Enfermeira, Doutora em Gerenciamento em Enfermagem. Bolsista do Programa Nacional de Pós-Doc da Universidade de São Paulo. São Paulo, SP, Brasil. E-mail: danielalourencao@usp.br.

2 Enfermeira, Doutora em Enfermagem. Professora Associada da Escola de Enfermagem da Universidade da Universidade de São Paulo. São Paulo, SP, Brasil. E-mail: daisyrt@usp.br.
}

Artigo recebido: 26/06/2017.

Artigo aprovado: 13/03/2018.

Artigo publicado: $26 / 07 / 2018$.

\section{Como citar esse artigo:}

Lourenção DCA, Tronchin DMR. Clima de segurança em centro cirúrgico: validação de um questionário para o cenário brasileiro. Rev. Eletr. Enf. [Internet]. 2018 [acesso em: ];20:v20a10. Disponível em: https://doi.org/10.5216/ree.v20.47570. 


\section{INTRODUÇÃO}

A segurança do paciente no Centro Cirúrgico (CC) engloba a complexidade das atividades desenvolvidas e a necessidade de trabalho em equipe no cenário cirúrgico, cuja característica envolve diferentes categorias, com identidades culturais distintas e atuando em um mesmo ambiente, com dinâmicas diversificadas de acordo com o modelo de gestão de cada instituição de saúde ${ }^{(1)}$.

Nesse contexto, embora os procedimentos cirúrgicos tenham a intenção de salvar vidas, as falhas no processo em determinadas situações podem causar danos irreparáveis ${ }^{(2)}$. Os Eventos Adversos (EA) perioperatórios representam uma taxa de $3 \%$ do total de procedimentos ao ano, evidenciando o impacto dos cuidados inseguros nos sistemas de saúde ${ }^{(3)}$.

A Organização Mundial da Saúde (OMS) identificou como o ponto mais crítico para a segurança cirúrgica a interação dos membros da própria equipe: cirurgiões, anestesiologistas, enfermeiros, dentre outros profissionais. A literatura ressalta que a cultura das equipes cirúrgicas são consideradas rígidas e resistentes às mudanças ${ }^{(3)}$.

Pesquisas vêm sendo conduzidas visando mensurar o clima de segurança percebido pelos profissionais, com vistas a avaliar a cultura de segurança nos serviços de saúde. A avaliação da segurança do paciente em ambiente cirúrgico implica considerar os aspectos relativos à cultura organizacional, ao clima de segurança do paciente e às peculiaridades inerentes ao processo de trabalho.

Em revisão de literatura acerca dos principais instrumentos de mensuração de cultura e clima de segurança com elevadas propriedades psicométricas e disponíveis por meio eletrônico ${ }^{(4-5)}$ destaca-se o Safety Attitudes Questionnaire (SAQ), desenvolvido por pesquisadores do Center of Excellence for Patient Safety Research and Practice, The University of Texas, sob a coordenação de Eric Thomas, John B. Sexton e Robert L. Heimreich ${ }^{(6)}$. Esse questionário mensura o clima de segurança nos serviços de saúde a partir da percepção dos profissionais acerca da segurança do paciente. Foi validado em seis estudos transversais, com uma amostra de 10.843 sujeitos, nos Estados Unidos da América (EUA), apresentando elevadas propriedades psicométricas nos seis domínios, a saber: clima de trabalho em equipe, satisfação no trabalho, percepção da gerência da unidade e do hospital, clima de segurança, condições de trabalho e percepção do estresse ${ }^{(7)}$.

O SAQ foi traduzido e validado em diversos países e em relação à confiabilidade do instrumento mensurada pelo teste Alpha de Cronbach, essa variou de 0,79 a 0,91 $91^{(7-11)}$. Os autores do questionário desenvolveram versões para diversos cenários como CC, Unidades de Terapia Intensiva (UTI), unidades ambulatoriais e atenção primária(612).

O Safety Attitudes Questionnaire/Operating Room (SAQ/OR) é a versão modificada do SAQ para o ambiente cirúrgico, que foi desenvolvida por Makary e equipe, sendo validada em um estudo, nos EUA, abrangendo 60 hospitais e 2.769 profissionais ${ }^{(13)}$. Os achados demonstraram consistência interna satisfatória, valor de alpha de Cronbach de 0,76 . Os estudos conduzidos pelos autores verificaram que as propriedades do instrumento, realmente, mensuram variáveis integrantes de clima de segurança em centro cirúrgico, com impacto na segurança do paciente ${ }^{(14)}$.

Considerando a lacuna de pesquisas e de instrumentos de mensuração de clima de segurança no ambiente de CC no Brasil, o SAQ/OR foi traduzido e adaptado para o referido contexto, segundo as etapas propostas na 
literatura ${ }^{(15)}$, originando o instrumento denominado $\mathrm{SAQ} / \mathrm{VCC}^{(1)}$. O presente estudo tem por objetivo avaliar as propriedades psicométricas da versão traduzida e adaptada do SAQ/OR para o contexto brasileiro.

\section{MÉTODO}

Trata-se de um estudo metodológico de validação das propriedades psicométricas do SAQ/OR, realizado posteriormente ao processo de tradução e adaptação cultural do questionário, fase esta, indispensável para garantir que a versão traduzida seja válida e confiável, para medir o que se propõe, tornando-se necessário testar essas duas propriedades ${ }^{(15)}$.

Para validar o instrumento traduzido e adaptado recorreu-se à análise da confiabilidade, por meio do alpha de Cronbach e a avaliação de constructo, com base na Análise Fatorial Exploratória (AFE), que foi adotada por ser um procedimento estatístico amplamente empregado no desenvolvimento e avaliação de instrumentos, cuja principal função é reduzir um grande número de variáveis observadas em um número de fatores que representam os constructos ou dimensões latentes ${ }^{(16)}$.

Nesta pesquisa, a AFE foi realizada utilizando-se o método por componentes principais e a rotação ortogonal VARIMAX. Cabe esclarecer que o critério empregado na seleção do número de fatores foi o de autovalores acima do valor um e como critérios para a exclusão dos itens, aqueles com valor de comunalidade inferior a 0,5 e os com carga fatorial inferior a 0,4 .

\section{Versão brasileira do SAQ/OR: Questionário de Atitudes de Segurança/Versão Centro Cirúrgico (SAQ/VCC)}

A versão original do SAQ/OR foi autorizada pelos idealizadores do questionário para a tradução e a validação para a língua portuguesa do Brasil. A tradução e adaptação transcultural produziu um instrumento denominado SAQ/VCC que manteve o mesmo layout, em duas páginas, do instrumento original(1).

Essa versão do SAQ/VCC permaneceu dividida em três partes, sendo a primeira referente à qualidade da comunicação e colaboração entre os profissionais atuantes em CC, onde o sujeito deve responder sobre a sua relação com cada uma das categorias profissionais elencadas; a segunda é composta por 58 assertivas que abarcam a segurança do paciente e um item questionando se a pesquisa foi respondida anteriormente e uma terceira composta pelas informações demográficas (gênero, raça/etnia, categoria profissional, tempo de atuação, turno de atuação, entre outros). Por fim, um espaço no qual é solicitado aos participantes, três recomendações para a melhoria da segurança do paciente em centro cirúrgico.

O questionário é respondido por meio de escala tipo Likert, com cinco graus, uma questão por escolha simples referente a ter respondido, anteriormente, o questionário e a solicitação de recomendações para a melhoria são abertas, para livre escolha dos participantes.

\section{Locais e participantes do estudo}

No presente estudo participaram quatro hospitais brasileiros, sendo dois privados e dois públicos e de ensino. A escolha das instituições deu-se em função do número de sujeitos pretendidos na amostra e da diversidade do perfil da população atendida nas instituições. Cabe salientar que as instituições privadas e uma 
pública são acreditadas por organizações internacionais. Os hospitais foram denominados pelas letras A, B, C e D, a fim de garantir o anonimato.

A amostra foi selecionada por conveniência e constituída por 590 profissionais de saúde atuantes nos CC, tendo como critérios de inclusão: enfermeiros, técnicos e auxiliares de enfermagem que trabalhassem no mínimo há seis meses na unidade e cirurgiões, residentes, anestesiologistas e instrumentadores que participassem ao menos de três procedimentos/mês.

\section{Procedimento de coleta de dados}

A coleta dos dados ocorreu no período de maio a agosto de 2014. Nas quatro instituições participantes foram realizadas reuniões com os diretores, gerentes e supervisores das equipes de profissionais dos CC, onde foram apresentados os objetivos e o método da pesquisa. Dependendo da dinâmica de trabalho, em algumas unidades o questionário e o Termo de Consentimento Livre e Esclarecido (TCLE) foram entregues aos gerentes de enfermagem ou coordenadores das equipes médicas e recolhido, posteriormente. Entretanto, como essa estratégia resultava em baixo retorno, os sujeitos foram abordados pessoalmente, aguardando o preenchimento do questionário no $\mathrm{CC}$ de cada instituição, majoritariamente, nas salas de conforto. O preenchimento do questionário levou aproximadamente 15 minutos.

\section{Análise dos dados}

Para a validação do estudo os resultados foram analisados considerando o total dos questionários respondidos nas instituições coparticipantes. Os dados coletados foram organizados em uma planilha eletrônica e a análise dos dados ocorreu por meio de recursos de computação de programa estatístico. As assertivas foram analisadas em relação aos atributos responsividade e aceitabilidade. A responsividade foi avaliada por meio dos efeitos chão (floor) e teto (ceiling); tais efeitos ocorrem quando mais que $15 \%$ das respostas estão concentradas no grau mínimo ou máximo da escala(17). A aceitabilidade foi verificada em função das respostas válidas e não válidas. Foram consideradas como não válidas: questões em branco, contendo mais de uma resposta ou aquelas assinaladas no item "não se aplica". Os resultados encontrados nos atributos responsividade e aceitabilidade permitiram produzir a AFE. Neste estudo o valor de Kaiser Meyer Olkin (KMO) foi de 0,844 e o de esfericidade de Bartlett de $p<0,001$, indicando que existem correlações suficientes entre as variáveis para prosseguir com a análise.

A validade de constructo foi avaliada empregando-se a AFE dos componentes principais e o método de rotação ortogonal VARIMAX ${ }^{(16)}$. Em seguida procedeu-se a análise da confiabilidade por meio do coeficiente de alpha de Cronbach, para a escala no total e para cada domínio/fator.

\section{Aspectos éticos}

O desenvolvimento do estudo atendeu as normas nacionais e internacionais de ética em pesquisa envolvendo seres humanos e foi aprovado pelo Comitê de Ética e Pesquisa (CEP) da Escola de Enfermagem da Universidade de São Paulo (EEUSP), CAAE - 19332613.4.0000.5392. Os participantes do estudo assinaram o TCLE em duas vias. 


\section{RESULTADOS}

O estudo resultou em uma amostra composta por $68,5 \%$ (cirurgiões, anestesiologistas, residentes de cirurgia e de anestesia) e a equipe de enfermagem (enfermeiros, técnicos de enfermagem e instrumentadores/circulantes) obteve o percentual de $31,2 \%$. Dos questionários respondidos no Hospital A foram obtidos 37\%, no Hospital B 17\%, no Hospital C 15\% e no Hospital D 31\%. Do total de 590 sujeitos 38,7\% eram cirurgiões/cirurgiões assistentes, $26,6 \%$ instrumentadores/circulantes de sala, $17,3 \%$ anestesiologistas/anestesiologistas assistentes e os residentes de cirurgia e de anestesia corresponderam a 17,4\%.

A maioria dos sujeitos do estudo $(55,7 \%)$ era do sexo masculino e da raça/etnia branca $(76,3 \%)$. A idade média foi de 39,62 anos (dp 10,8) e a mediana de 36,32 anos. Os dados mostraram que a média de experiência de trabalho na especialidade foi de 13,41 anos $(\mathrm{dp} 10,7)$ e em relação à média de tempo de trabalho na instituição correspondeu a 8,93 anos (dp 9,2).

\section{Propriedades psicométricas: análise fatorial exploratória}

A AFE inicial apontou a existência de 17 fatores que explicavam 66,1\% da variância total dos 58 itens positivos e negativos. Nas análises fatoriais subsequentes foram eliminados os itens cujas cargas fatoriais e/ou comunalidade eram inferiores aos critérios pré-estabelecidos. Os itens eliminados em cada etapa são apresentados na Tabela 1 e os valores em negrito apontam o motivo da exclusão, totalizando 18 itens.

Tabela 1: Distribuição dos itens eliminados e respectivas cargas fatoriais e comunalidades. São Paulo, SP, Brasil, 2014.

\begin{tabular}{|c|c|c|c|}
\hline Etapa & Item eliminado & $\begin{array}{c}\text { Carga } \\
\text { fatorial }\end{array}$ & Comunalidade \\
\hline 1 & 33. (N)* Estresse devido a problemas pessoais afeta de forma adversa o meu desempenho. & 0,312 & 0,691 \\
\hline 2 & 30. Neste Centro Cirúrgico, as discordâncias são resolvidas de modo apropriado. & 0,326 & 0,611 \\
\hline 3 & 34. Eu tenho apoio que necessito de outros membros da equipe para cuidar dos pacientes. & 0,341 & 0,574 \\
\hline 4 & $\begin{array}{l}\text { 55. Durante situações de emergência, meu desempenho não é afetado ao trabalhar com } \\
\text { pessoal inexperiente ou menos capacitado. }\end{array}$ & 0,352 & 0,594 \\
\hline 5 & 39. (N) Frequentemente, não consigo expressar desacordo com a equipe médica. & 0,376 & 0,636 \\
\hline 6 & $\begin{array}{l}\text { 3. As sugestões do enfermeiro sobre a assistência ao paciente são bem recebidas no Centro } \\
\text { Cirúrgico. }\end{array}$ & 0,352 & 0,576 \\
\hline 7 & 6. Este hospital faz um bom trabalho no treinamento de novos membros da equipe. & 0,337 & 0,489 \\
\hline 8 & 42. Estagiários da minha área são supervisionados adequadamente. & 0,306 & 0,438 \\
\hline 9 & $\begin{array}{l}\text { 38. Os médicos e os enfermeiros daqui trabalham em conjunto como uma equipe bem } \\
\text { coordenada. }\end{array}$ & 0,327 & 0,563 \\
\hline 10 & $\begin{array}{l}\text { 27. (N) Eu tenho visto outros profissionais cometerem erros que tinham potencial de } \\
\text { prejudicar os pacientes. }\end{array}$ & 0,354 & 0,572 \\
\hline 11 & $\begin{array}{l}\text { 37. Durante as situações de emergências, posso prever o que outros profissionais farão em } \\
\text { seguida. }\end{array}$ & 0,430 & 0,482 \\
\hline 12 & $\begin{array}{l}\text { 43. Conheço o nome e sobrenome completo de todo o pessoal com quem trabalhei no meu } \\
\text { último turno. }\end{array}$ & 0,366 & 0,598 \\
\hline 13 & $\begin{array}{l}\text { 56. (N) Os profissionais, frequentemente, não levam em consideração as regras ou diretrizes } \\
\text { estabelecidas neste Centro Cirúrgico. }\end{array}$ & 0,370 & 0,594 \\
\hline 14 & 1. (N) Neste Centro Cirúrgico são comuns altos níveis de carga de trabalho. & 0,357 & 0,607 \\
\hline 15 & 29. Eu me orgulho de trabalhar neste hospital & 0,381 & 0,640 \\
\hline 16 & 8. Trabalhar neste hospital é como fazer parte de uma grande família. & 0,521 & 0,497 \\
\hline 17 & $\begin{array}{l}\text { 36. (N) Interrupções na continuidade do atendimento podem ser prejudiciais para a } \\
\text { segurança do paciente. }\end{array}$ & $-0,375$ & 0,575 \\
\hline 18 & 44. (N) Cometi erros que poderiam prejudicar os pacientes. & 0,464 & 0,493 \\
\hline
\end{tabular}

Legenda: * $(\mathrm{N})$ : Assertivas negativas / *Negrito: valores de exclusão. 
O SAQ/VCC apresentou uma escala multidimensional e a AFE apontou a exclusão de 18 itens, assim, os 40 itens restantes foram distribuídos em 12 fatores/domínios que explicavam 64,3\% da variância e foram submetidos ao teste do alpha de Cronbach. Desses itens 30 foram organizados nos seis primeiros fatores/domínios e apresentaram o alpha maior que 0,50 e seis itens obtiveram valores inferiores e o número de itens variou de um a três em cada fator e não foram considerados como domínios. Desse modo, das 40 assertivas do questionário 30 foram alocadas nos domínios e 10 permaneceram nos fatores.

Dos 12 fatores encontrados na AFE foram definidos como domínios os seis primeiros, consonantes às outras versões do $S A Q$, a saber: clima de segurança, percepção da gerência, percepção do estresse, condição do trabalho, comunicação no ambiente cirúrgico, percepção do desempenho profissional. A escolha da nomenclatura dos domínios foi realizada pelas pesquisadoras, com base na revisão de literatura, na semântica dos itens e nos estudos sobre adaptação cultural do SAQ para os diversos países e nos estudos internacionais empregando o SAQ/OR para avaliar clima de segurança. Os itens referentes aos domínios são apresentados na Tabela 2.

Tabela 2: Descrição dos itens de cada domínio, correlação item-total e alpha de Cronbach se o item for excluído. São Paulo, SP, Brasil, 2014.

\begin{tabular}{|c|c|c|}
\hline & $\begin{array}{l}\text { Correlação Item } \\
\text { - Total }\end{array}$ & $\begin{array}{l}\text { Alpha de Cronbach se } \\
\text { o item for excluído }\end{array}$ \\
\hline \multicolumn{3}{|l|}{ Clima de Segurança (Alpha de Cronbach = 0,82) } \\
\hline $\begin{array}{l}\text { 46. Todo o pessoal do Centro Cirúrgico assume responsabilidade pela segurança do } \\
\text { paciente. }\end{array}$ & 0,62 & 0,79 \\
\hline $\begin{array}{l}\text { 51. Aqui existe adesão ampla às diretrizes clínicas e critérios baseados em } \\
\text { evidências relacionados com segurança do paciente. }\end{array}$ & 0,64 & 0,79 \\
\hline $\begin{array}{l}\text { 54. As informações obtidas por meio dos relatórios de eventos adversos são usadas } \\
\text { para tornar a assistência do paciente mais segura neste Centro Cirúrgico. }\end{array}$ & 0,54 & 0,80 \\
\hline 50. Assuntos importantes são bem comunicados nas mudanças de turno. & 0,55 & 0,80 \\
\hline $\begin{array}{l}\text { 48. A segurança do paciente é, constantemente, reforçada como prioridade aqui no } \\
\text { Centro Cirúrgico. }\end{array}$ & 0,60 & 0,79 \\
\hline 41. O moral neste Centro Cirúrgico é alto & 0,54 & 0,80 \\
\hline 45. A equipe médica deste Centro Cirúrgico faz um bom trabalho. & 0,47 & 0,81 \\
\hline \multicolumn{3}{|l|}{ Percepção da Gerência (Alpha de Cronbach $=0,79$ ) } \\
\hline 10. A administração deste hospital apoia meus esforços diários. & 0,73 & 0,69 \\
\hline 9. A administração deste hospital está fazendo um bom trabalho. & 0,69 & 0,71 \\
\hline 11. Eu recebo retorno apropriado sobre o meu desempenho. & 0,59 & 0,74 \\
\hline 15. Este hospital é um bom lugar para se trabalhar. & 0,46 & 0,79 \\
\hline $\begin{array}{l}\text { 18. Neste Centro Cirúrgico o número de profissionais é suficiente para atender o } \\
\text { número de pacientes. }\end{array}$ & 0,43 & 0,81 \\
\hline \multicolumn{3}{|l|}{ Percepção do Estresse (Alpha de Cronbach $=0,78$ ) } \\
\hline $\begin{array}{l}\text { 47. }(N) * \text { Sinto-me cansado quando levanto pela manhã e tenho que enfrentar outro } \\
\text { dia de trabalho. }\end{array}$ & 0,66 & 0,69 \\
\hline 49. (N) Eu me sinto exausto com o meu trabalho. & 0,65 & 0,70 \\
\hline 53. (N) Sinto que estou trabalhando demais. & 0,57 & 0,74 \\
\hline 52. (N) Sinto-me frustrado com o meu trabalho. & 0,50 & 0,77 \\
\hline \multicolumn{3}{|l|}{ Condição do Trabalho (Alpha de Cronbach $=0,77$ ) } \\
\hline $\begin{array}{l}\text { 21. A cultura de segurança neste Centro Cirúrgico torna fácil aprender com os erros } \\
\text { dos outros. }\end{array}$ & 0,59 & 0,72 \\
\hline $\begin{array}{l}\text { 28. Eu conheço os meios adequados para encaminhar questões relacionadas à } \\
\text { segurança do paciente neste Centro Cirúrgico. }\end{array}$ & 0,50 & 0,75 \\
\hline $\begin{array}{l}\text { 26. Recebo informações adequadas e oportunas sobre ocorrências no hospital, que } \\
\text { podem afetar o meu trabalho. }\end{array}$ & 0,53 & 0,74 \\
\hline
\end{tabular}


Correlação Item Alpha de Cronbach se

- Total o item for excluído

22. O hospital lida de maneira construtiva com os profissionais problemáticos.

$\begin{array}{ll}0,47 & 0,75 \\ 0,51 & 0,74 \\ 0,51 & 0,74 \\ 0,36 & 0,54 \\ 0,44 & 0,47 \\ 0,29 & 0,59 \\ 0,46 & 0,45 \\ 0,65 & 0,56 \\ 0,55 & 0,63 \\ 0,43 & 0,70 \\ 0,40 & 0,72\end{array}$

35. É fácil para os profissionais que atuam neste Centro Cirúrgico fazerem perguntas quando existe algo que não entendem.

20. Sou encorajado por meus colegas a informar qualquer preocupação que eu possa ter com a segurança do paciente.

0,74

0,74

Comunicação no ambiente cirúrgico (Alpha de Cronbach $=0,59$ )

23. Os equipamentos deste Centro Cirúrgico são adequados.

19. A tomada de decisão no Centro Cirúrgico utiliza informações dos profissionais envolvidos.

13. A transmissão de informações entre os profissionais do Centro Cirúrgico antes da realização de um procedimento cirúrgico é importante para a segurança do paciente.

14. A transmissão de informações é comum no Centro Cirúrgico.

Percepção do desemprenho profissional (Alpha de Cronbach $=\mathbf{0 , 7 1}$ )

31. (N) Sou menos eficiente no trabalho quando estou cansado.

25. (N) Quando a minha carga de trabalho se torna excessiva, meu desempenho é prejudicado.

16. (N) O cansaço prejudica meu desempenho durante situações de emergência.

32. (N) Eu tenho maior probabilidade de cometer erros em situações tensas ou hostis.

Legenda: $(\mathrm{N})$ : Assertivas negativas.

\section{Análise da confiabilidade do questionário}

A análise de confiabilidade dos 40 itens do questionário SAQ/VCC foi realizada por meio da consistência interna, empregando-se o coeficiente de alpha de Cronbach, que apresentou o valor geral de 0,87 e nos domínios variaram de 0,59 a 0,82, demonstrando a confiabilidade do instrumento (Tabela 3).

Tabela 3: Descrição do valor de alpha de Cronbach geral e dos domínios do SAQ/VCC. São Paulo, SP, Brasil, 2014.

\begin{tabular}{lcc}
\hline \multicolumn{1}{c}{ Domínios do SAQ/VCC* } & Número de itens & Alpha de Cronbach \\
\hline SAQ Total & 40 & 0,87 \\
1. Clima de Segurança & 7 & 0,82 \\
2. Percepção da Gerência & 5 & 0,79 \\
3. Percepção do Estresse & 4 & 0,78 \\
4. Condição de Trabalho & 6 & 0,77 \\
5. Comunicação no ambiente cirúrgico & 4 & 0,59 \\
6. Percepção do desempenho profissional & 4 & 0,71 \\
\hline
\end{tabular}

Legenda: * SAQ/VCC - Questionário de Atitudes de Segurança/Versão Centro Cirúrgico.

Pela análise das propriedades psicométricas os fatores que apresentaram o alpha de Cronbach menor que 0,50, não foram nominados como domínios específicos de um constructo. Assim, esses itens foram agrupados em fatores numerados como sete, oito, nove, 10, 11 e 12, perfazendo um total de 10 assertivas e merecem ser reavaliados, com número maior de sujeitos para determinar se podem ser incorporados em algum dos domínios.

Dessa forma, os 40 itens resultantes deste estudo produziram a versão reduzida do questionário, que foi denominada Questionário de Atitudes de Segurança/Centro Cirúrgico - SAQ/CC.

Os achados da presente pesquisa demonstraram a avaliação positiva e a confiabilidade do instrumento, que apresentou o alpha de Cronbach de 0,87, para as 40 assertivas definidas pela AFE. Os seis domínios deste estudo apresentaram uma variação do alpha de 0,59 a 0,82. O menor valor foi representado pelo domínio comunicação no ambiente cirúrgico composto por quatro itens. 


\section{DISCUSSÃO}

Este estudo representa a primeira validação da versão adaptada para o Brasil do questionário que mensura clima de segurança em ambiente cirúrgico. Baseado em nossos resultados a consistência interna e a validação de constructo foram avaliados e produziram os domínios extraídos da Análise Fatorial Exploratória (AFE). Pesquisas demonstraram que os resultados da consistência interna deste estudo são correlatos aos estudos conduzidos na Suécia, em Portugal e no Japão(18-20).

Os estudos realizados aplicando o SAQ em sua versão genérica apresentam seis domínios: clima de trabalho em equipe, satisfação no trabalho, percepção da gerência, clima de segurança, condições de trabalho e percepção do estresse ${ }^{(6)}$. Dentre os que adaptaram o SAQ/OR nos três países supracitados, não foram explicitados os domínios específicos da versão CC, apropriando-se dos domínios da versão genérica ${ }^{(18-20)}$. Por outro lado, os estudos destinados a mensurar o clima de segurança com o SAQ/OR utilizaram domínios da versão genérica, desconsiderando os itens específicos de CC para comporem outros domínios ${ }^{(21-22)}$. Esses achados evidenciam a necessidade em se empregar o questionário específico de acordo com o ambiente/área, assim como os respectivos domínios para avaliação do clima de segurança em ambiente cirúrgico.

Pela AFE foi possível identificar o constructo latente às questões do questionário adaptado para o contexto brasileiro, especialmente, em relação à sua dimensionalidade, contribuindo para a elaboração dos domínios do $\mathrm{SAQ} / \mathrm{CC}$.

Conforme descrito anteriormente, os domínios deste estudo foram: clima de segurança, percepção da gerência, percepção do estresse, condição de trabalho, comunicação no ambiente cirúrgico e percepção do desempenho profissional. Assim, os quatro primeiros domínios são semelhantes aos outros estudos do $S A Q$, contudo o quarto e o quinto domínio foram nominados pelas pesquisadoras, tendo em vista que os itens se referem ao ambiente cirúrgico e diferem dos domínios do SAQ - versão genérica. O domínio clima de segurança indica a percepção dos profissionais quanto ao comprometimento organizacional proativo para segurança do paciente ${ }^{(7,13,23)}$. O segundo domínio, descrito como percepção da gerência, diz respeito à aprovação das ações da gerência quanto às questões de segurança. Em relação ao terceiro domínio, percepção do estresse, esse aborda o reconhecimento do quanto os fatores estressores influenciam na execução do trabalho. O quarto domínio refere-se à condição de trabalho e relaciona-se com a percepção da qualidade do ambiente de trabalho e no nosso estudo os itens, apontados pela análise fatorial, remetem às questões relacionadas com a segurança do paciente na organização do cotidiano profissional.

Pelos achados da investigação, o quinto domínio foi identificado como comunicação no ambiente cirúrgico. Trata-se de um domínio específico para o ambiente cirúrgico, pois seus itens referem-se à informação compartilhada entre os profissionais de saúde que interferem na segurança do paciente.

Sob esse prisma, a literatura destaca a responsabilidade do enfermeiro em estabelecer um sistema de comunicação que abarque os recursos materiais e de equipamentos e a equipe cirúrgica para garantir um ambiente seguro para o paciente e também para os profissionais que atuam nesse ambiente ${ }^{(24)}$. Ressalta-se que os procedimentos cirúrgicos requerem o emprego do equipamento correto e em boas condições de funcionamento e as medicações têm de ser administradas no tempo adequado. 
Dessa forma, como os papéis das categorias profissionais que atuam no ambiente cirúrgico são interdependentes, cabe à equipe de anestesiologistas, de enfermagem e de cirurgiões se comunicarem de forma eficaz para prevenir complicações evitáveis ${ }^{(25)}$.

Os autores do questionário original propuseram a mensuração da comunicação e da colaboração entre as categorias profissionais que atuam nesse ambiente, como a primeira parte do questionário(7).

Assim, ressalta-se a contribuição desta pesquisa ao desenvolvermos um domínio que mensura esse constructo e permite avaliar as tomadas de decisões baseadas na comunicação no ambiente cirúrgico. Por conseguinte, esse domínio possibilita a análise de um aspecto crucial do processo de trabalho das equipes cirúrgicas, ao relacionar os dados da primeira parte do questionário com os resultados deste domínio, o que permitirá adensar a avaliação da comunicação e da colaboração entre os profissionais de saúde que atuam nesse ambiente.

E, finalmente, o sexto domínio, que também foi definido e nominado neste estudo, percepção do desempenho profissional. Dessa maneira, ao analisarmos a semântica dos itens do sexto domínio observa-se que os mesmos referem-se à repercussão do cansaço e da sobrecarga de trabalho no desempenho profissional. Acreditamos que um aspecto fundamental para a segurança do paciente é a capacidade individual de reconhecer e assumir o cansaço e o quanto esse fator afeta a atuação profissional. Observamos que os itens agrupados neste domínio encontram-se nos estudos da versão short form do SAQ original e da versão adaptada para o português, no domínio reconhecimento/percepção do estresse ${ }^{(7,23)}$.

Os estudos de adaptação e validação do $S A Q / O R$, desenvolvidos nos países Suécia, Portugal e Japão, realizaram o processo de tradução e adaptação conforme a metodologia internacional recomenda. Todavia, na validação analisaram o alpha de Cronbach total, mas não analisaram os domínios ou analisaram apenas alguns itens ou alguns domínios da versão genérica(18-20). Por esse motivo, não há estudos para compararmos os domínios encontrados na nossa análise fatorial exploratória. Nessa direção, um estudo realizado em um centro de cirurgia cardíaca pediátrica, analisou os itens que compõe o $S A Q / O R$ desconsiderando os domínios ${ }^{(21)}$ e, ainda, a pesquisa conduzida no CC do Veterans Health Administration (VHA) aplicou o SAQ/OR analisando apenas os itens do domínio clima de segurança(22).

A confiabilidade do SAQ/CC é semelhante a de outros estudos de adaptação transcultural do SAQ/OR. Entretanto, torna-se necessário ampliar a pesquisa em outros cenários cirúrgicos brasileiros para consolidar as propriedades psicométricas.

\section{Limitação do estudo}

Entendemos como limitação deste estudo a baixa adesão da equipe de enfermagem e a inexistência de estudos na literatura contendo os itens dos domínios referentes ao $S A Q$ versão CC, o que impossibilitou a comparação das propriedades psicométricas relativas aos domínios.

Nesse sentido, consideramos que novas pesquisas devem ser desenvolvidas, empregando-se amostras com distribuição homogênea entre as categorias profissionais e outras análises, como a análise fatorial confirmatória, visando confirmar a estrutura fatorial do questionário. 


\section{CONCLUSÃO}

A versão brasileira do $S A Q / O R$ tem validade de constructo satisfatória e o instrumento mostrou-se confiável apresentando o valor geral de alpha de Cronbach de 0,87 e nos domínios variaram de 0,59 a 0,82. Os domínios elencados neste estudo foram: clima de segurança, percepção da gerência, percepção do estresse, condição de trabalho, comunicação no ambiente cirúrgico e percepção do desempenho profissional.

A versão produzida por este estudo, denominada SAQ/CC, poderá facilitar a reprodução da pesquisa em outros cenários cirúrgicos do Brasil, em virtude de aprimorar a coleta de dados e a adesão dos profissionais.

Esta pesquisa permitiu validar um instrumento que avalia o clima de segurança no $\mathrm{CC}$, considerado um ambiente vulnerável à ocorrência de eventos que comprometem a segurança do paciente, sobretudo decorrente da necessidade da comunicação efetiva entre os profissionais de saúde e dos riscos inerentes ao procedimento cirúrgico.

\section{REFERÊNCIAS}

1. Lourenção DCA, Tronchin DMR. Segurança do paciente no ambiente cirúrgico: tradução e adaptação cultural de instrumento validado. Acta paul. enferm. [Internet]. 2016 Fev [acesso em: 12 Nov 2017] ; 29( 1): 1-8. Disponível em: http://www.scielo.br/scielo.php?script=sci_arttext\&pid=S0103-21002016000100002\&lng=pt. http://dx.doi.org/10.1590/19820194201600002.

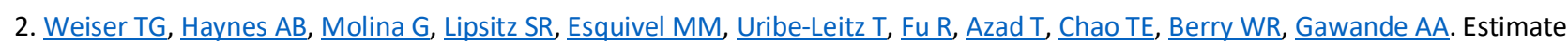
of the global volume of surgery in 2012: an assessment supporting improved health outcomes. Lancet [Internet]. 2015 [acesso em:20 Jan 2016]; Apr 27;385 Suppl 2:S11. Disponível em:

http://www.thelancet.com/journals/lancet/article/PIIS0140-6736(15)60806-6/fulltext

3. Organização Mundial da Saúde. Segundo desafio global para a segurança do paciente: Cirurgias seguras salvam vidas (orientações para cirurgia segura da OMS). Trad. de Marcela Sánchez Nilo e Irma Angélica Durán. Rio de Janeiro: Organização PanAmericana da Saúde; Ministério da Saúde; Agência Nacional de Vigilância Sanitária, 2009. 211 p. . [acesso em:25 Jan 2013]. Disponível em: http://bvsms.saude.gov.br/bvs/publicacoes/seguranca paciente cirurgias seguras salvam vidas.pdf 4. The Health Foundation. Evidence Center. Measuring safety culture. London: The Health Foundation [Internet]. 2011. [acesso em:25 Jan 2013]. Disponível em: http://www.health.org.uk/publications/measuring-safety-culture/.

5. Colla JB, Bracken AC, Kinney LM, Weeks WB. Measuring patient safety climate: a review of surveys. Qual Saf Health Care [Internet]. 2005;14(5):364-366. [acesso em:25 Jan 2013]. Disponível em:

https://www.ncbi.nlm.nih.gov/pmc/articles/PMC1744072/pdf/v014p00364.pdf

6. Sexton JB, Thomas EJ. The Safety Attitude Questionnaire (SAQ) Guidelines for Administration. Technical Report 03-02. The University of Texas Center of Excellence for Patient Safety Research and Practice [Internet]. 2003 [acesso em:10 Jan 2013]. Disponível em: http://www.nationalpatientsafetyfoundation.org/wp-

content/uploads/2011/10/RG SUPS Sharing Mod3 Sexton.pdf.

7. Sexton, J.B, Helmreich RL, Neilands TB, Rowan K, Vella K, Boyden J, et al. The Safety Attitudes Questionnaire: Psychometric Properties, Benchmarking Data, and Emerging Research. BMC Health Serv Res. [Internet]. 2006 [acesso em:10 Jan 2013];6: $44-54$. Disponível em: http://www.ncbi.nlm.nih.gov/pmc/articles/PMC1481614/

8. Deikås E, Hofoss D. Psychometric properties of the Norwegian version of the Safety Attitudes Questionnaire (SAQ), Generic version (short form 2006). BMC Health Serv Res [Internet]. 2008 [acesso em: 29 Jan2013];8:191-198. Disponível em: http://www.orgsun.com/1/18/62634-1-operating-room-version-safety-attitudes-questionnaire-analysis-using.php.

9. Kaya S, Barsbay S, Karabulut E. The Turkish Version of the safety attitude questionnaire: psychometric properties and baseline data. Qual Saf Health Care. [Internet]. 2010 [acesso em: 28 Jan 2013]; 19:572-577. Disponível em:

http://www.ncbi.nlm.nih.gov/pubmed/20671082.

10. Lee WC, Wung HY, Liao HH, Lo CM, Chang FL, Wang PC, et al. Hospital safety culture in Taiwan: A nationwide survey using chinese version safety attitude questionnaire. BMC Health Serv Res. [Internet]. 2010 [acesso em: 28 Jan 2013]; 10: $234-242$. Disponível em: http://www.biomedcentral.com/1472-6963/10/234.

11. Nordén-Hägg A, Sexton JB, Kälvernark-Sporrong S, Ring L, Kettis-Lindblad A. Assessing safety culture in Pharmacies: The psychometrics validation of the Safety Attitudes Questionnaire (SAQ) in a national sample of community pharmacies in Sweden. BMC Clin Pharmacol. [Internet]. 2010 [acesso em: 28 Jan 2013];10:8-20. Disponível em: http://www.biomedcentral.com/1472$\underline{6904 / 10 / 8 .}$ 
12. Kristensen S, Sabroe S, Bartels P, Mainz J, Christensen KB. Adaption and validation of the Safety Attitudes Questionnaire for the Danish hospital setting. Clin Epidemiol [Internet]. 2015 [acesso em: 26 jul. 2018];7:149-60. Disponível em:

https://doi.org/10.2147/CLEP.S75560.

13. Makary, MA; Sexton JB, Freischlag JA, Millman A, Pryor D, Holzmueller C, Pronovost PJ. Patient Safety in Surgery. Ann Surg. [Internet]. 2006 [acesso em: 21 Jan 2013]. May; 243(5): 628-635. Disponível em:

http://www.ncbi.nlm.nih.gov/pmc/articles/PMC1570547/.

14. Makary, MA, Sexton, JB, Freischlag, JA, Holzmueller, CG, Millman, A, Rowen, L, Pronovost, PJ. Operating Room Teamwork among Physicians and Nurses: Teamwork in the Eye of the Beholder. J Am Coll Surg. 2006 [acesso em: 21 Jan 2013]; May

202(5):746-52. Disponível em: http://www.ncbi.nlm.nih.gov/pubmed/16648014

15. Beaton DE, Bombardier C, Guillemin F, Ferraz MB. Guidelines for the Process of Cross-Cultural Adaptation of Self-Report

Measures. SPINE [Internet]. 2000 [acesso em:08 Mar 2013];25(24):3186-319. Disponível em:

http://www.ncbi.nlm.nih.gov/pubmed/11124735.

16. Hair JF Jr, Black WC, Babin BJ, Anderson RE, Tatham RL. Análise multivariada de dados. Trad. de Adonai Sclup Sant"Anna. 6aed. Porto Alegre: Bookman; 2009.

17. Terwee CB, Bot SDM, Boer MR, van der Windt DAWM, Knol DL, Dekker J, et al.. Quality criteria were proposed for measurement properties of health status questionnaires. J Clin Epidemiol [Internet]. 2007 [acesso em:25 Fev 2015]; 60:34-42. Disponível em: http://www.ncbi.nlm.nih.gov/pubmed/17161752.

18. Göras C, Wallentin FY, Nilsson U, Ehrenberg A. Swedish translation and psychometric testing of the safety attitudes questionnaire (operation room version). BMC Health Serv Res. [Internet]. 2013 [acesso em: 28 Jan 2013]; 13: 104 - 111. Disponível em: http://www.biomedcentral.com/content/pdf/1472-6963-13-104.pdf.

19. Pinheiro JPA, Uva AS. Safety climate in the operating room: Translation, validation and application of the Safety Attitudes Questionnaire. Rev Port Saúde Pública [Internet]. 2016 [acesso em:10 Out 2017]; 34(2): 107-116. Disponível em:

https://www.sciencedirect.com/science/article/pii/S0870902515000474.

20. Kawano T, Taniwaki M, Ogata K, Sakamoto M, Yokoyama M. Improvement of teamwork and safety climate following implementation of the WHO surgical safety checklist at a university hospital in Japan. J Anesth [Internet]. 2014 [acesso em:10 Jan 2015]; 28:467-470. Disponível em: http://www.ncbi.nlm.nih.gov/pubmed/24170220.

21. Bognár A, Barach P, Julie K, Johnson MSPH, Duncan RC, Birnbach D, et al.. Errors and the Burden of Errors: Attitudes, Perceptions, and the Culture of Safety in Pediatric Cardiac Surgical Teams. Ann Thorac Surg [Internet]. 2008 [acesso em: 20 Nov 2014];85:1374 - 81. Disponível em: http://www.annalsthoracicsurgery.org/article/S0003-4975(07)02358-2/pdf.

22. Carney BT, Mills PD, Bagian JP, Weeks WB. Sex differences in operating room care giver perceptions of patient safety: a pilot study from the Veterans Health Administration Medical Team Training Program. Qaul Saf Health Care [Internet]. 2010 [acesso em: 07 Oct 2014];19: 128-131. Disponível em: http://www.ncbi.nlm.nih.gov/pubmed/20142406.

23. Carvalho REFL, Cassiani SHB. Cross-cultural adaptation of the Safety Attitudes Questionnaire - Short Form 2006 for Brazil. Rev Lat Am Enfermagem. [Internet]. 2012 [acesso em: 15 Jan 2014];20(3). Disponível em:

http://www.scielo.br/pdf/rlae/v20n3/pt a20v20n3.pdf

24. Silva DC, Alvim NAT. Surgical Center environment and its elements: implications for nursing care. Rev Bras Enferm. [Internet]. 2010[acesso em: 10 Oct 2014]; 63(3): 427-34. Disponível em: http://www.scielo.br/pdf/reben/v63n3/a13v63n3.pdf 25. Pugel AE, Simianu VV, Flum DR, Dellinger EP. Use of the surgical safety checklist to improve communication and reduce complications. On line J Infect Public Health [Internet] 2015 [acesso em: 04 Mar 2015]; 397:1-7. Disponível em:

http://dx.doi.org/10.1016/i.jiph.2015.01.001 